\title{
PARASITISMO ARGUMENTAL: EL PUNTO MUERTO DE LA DELIBERACIÓN
}

\author{
Argumental Parasitism: The Deadlock for Deliberation \\ María Luján Christiansen \\ Universidad de Guanajuato \\ mlchris_mex@hotmail.com
}

\begin{abstract}
Resumen:
Dentro del campo disciplinar de la filosofía, la argumentación ha sido considerada como un tema esencialmente lógico-analítico. No obstante, tal abordaje no proporciona una comprensión de la práctica argumental tal como se despliega en las situaciones de conflicto. Desde una perspectiva pragmática, es posible identificar argumentaciones formalmente correctas que, sin embargo, inducen a convencimientos forzados y violencias epistemológicas. En este artículo, se toman como foco de análisis crítico las denominadas "racionalizaciones vertiginosas", las cuales se sustentan en una lógica epistemicida criterial que termina siendo parasitaria para la toma de decisiones. Las mismas contrastan significativamente con procesos argumentativos planteados desde un pluralismo cognitivo que acepta la incertidumbre, la informalidad y la subdeterminación de los argumentos como parte constitutiva de los encuentros dialógicos reales. Se propone que, en tanto condición de posibilidad de la deliberación y la acción colectiva, la argumentación es un proceso regido por reglas, pero también por virtudes cultivables en una cultura auténticamente democrática.
\end{abstract}

\section{Palabras clave:}

Argumentación, Epistemicidio, Vértigo, Parasitismo, Virtuosismo

\begin{abstract}
:
Within the disciplinary field of philosophy, argumentation has been considered as an essentially logical-analytical issue. However, such an approach does not provide an understanding of the argumental practice as it unfolds in conflict situations. From a pragmatic perspective, it is possible to identify formally correct arguments that, however, induce forced convictions and epistemological violence. In this article, the so-called "vertiginous rationalizations" are taken as the focus of critical analysis, which are based on a criterial epistemicida/ logic that ends up being parasitic for decision making. They contrast significantly with argumentative processes raised from a
\end{abstract}


cognitive pluralism that accepts uncertainty, informality and underdetermination of arguments as a constitutive part of real dialogic encounters. It is proposed that, as a condition for the possibility of deliberation and collective action, argumentation is a process governed by rules, but also by cultivable virtues in a truly democratic culture.

\section{Keywords:}

Argumentation, Epistemicide, Vertigo, Parasitism, Virtuosity

Recibido: 29/09/2019

Aceptado: 20/11/2019

"Anular las otras razones es el primer paso para intentar anular al otro, al otro en los demás y al otro en uno mismo"

Carlos Pereda (1994: 15)

\section{INTRODUCCIÓN}

¿Qué es argumentar? Desde una fría perspectiva lógico-analítica, la argumentación puede entenderse como un estudio de las conexiones entre enunciados o como un tipo específico de acto de habla. Sin embargo, tal definición resulta totalmente insuficiente para aprehender la argumentación como una experiencia interpersonal. Si el acto de argumentar se redujera meramente a evaluar la validez o invalidez de las creencias en el sentido de la lógica formal, no podría entenderse la ebullición de emociones que se ponen en juego durante una acalorada discusión en la que todas las partes quieren tener razón. Pierre Bourdieu acertó en señalar este punto, al afirmar que la lógica de la práctica difiere de la lógica de los lógicos (cit. por Illouz, 2009: 244) y que, como sentenciaba también el perspicaz filósofo Carlos Pereda , un argumento puede "revelar" u "ocultar", puede ser "sagaz" o "miope", "penetrante" o "superficial", "justo" o "distorsionador", "con mucho tacto" o "casi sin tacto", "abridor de caminos" u "opresivo", "sutil con el problema que trata" o "brutal", o, incluso, "en parte, sutil" y "en parte, brutal" (Pereda, 1994: 11).

Por lo tanto, la pregunta "¿qué hace que un argumento sea un buen argumento?" se queda muy corta si pretendemos comprender la argumentación como algo más que la consistencia enunciativa. En su dimensión pragmática, la argumentación tiene un costado vincular-relacional ineludible: ¿qué significa el aceptar no tener razón? ¿Quién es quién dentro de un debate? ¿Qué se espera ganar cuando se busca desesperadamente convencer al Otro? ¿Qué se pierde cuando se pierde? ¿Para qué argumenta el que 
argumenta? Y, finalmente, el interrogante crucial para el presente artículo: al argumentar, ¿se busca inexorablemente un camino hacia la deliberación?

\section{ARGUMENTAR: ¿DAR RAZONES O "EMBARRAR LA CANCHA"?}

El caldo de cultivo para la práctica de la argumentación son las diferencias, en especial cuando son opuestas: dondequiera que aparezcan creencias antagónicas, emergen condiciones para querer defender las ideas propias o atacar las del otro. Las creencias diferentes no necesariamente tienen que entrar en conflicto, pero, cuando ocurre, hay básicamente, dos estilos de afrontamiento: negar el conflicto (evitación) o resolverlo. Si se sigue la segunda vía (de querer solucionar el problema), hay dos estrategias principales: imponerse por la fuerza o por la argumentación. A partir de aquí, parece inferirse que el uso de la fuerza es una forma violenta de resolución del conflicto, mientras que hacerlo mediante la argumentación es una manera mucho más racional, respetuosa y madura de abordar las diferencias. Dicho brevemente, la argumentación se presenta como antitética a la violencia: donde hay una, no está la otra.

Ahora, si esta última afirmación se generaliza, constituye un error grave, ya que hay formas de argumentación que no se orientan en lo más mínimo a la clarificación, la comprensión, la deliberación y la acción colectiva, sino a la autovalidación epistémica, la reafirmación omnipotente y vanidosa del propio punto de vista y la legitimación de la superioridad dentro de la relación interpersonal. Tal posicionamiento dentro del terreno conversacional constituye una forma de violencia, la cual puede ser caracterizada como una violencia simbólica invisibilizada, que opera desde el interior de la práctica misma (a diferencia de la violencia externa, en la cual las razones del otro son aniquiladas mediante la amenaza, la manipulación, la agresión física, el chantaje, la seducción u otra modalidad).

¿En qué escenarios se vuelve posible que la argumentación aumente la violencia, en lugar de frenarla? Ello ocurre, frecuentemente, cuando las argumentaciones tienen ciertas peculiaridades, independientemente de que el argumentador lo sepa o no. Hay que recordar que, en el momento en que "da razones", el argumentador no reflexiona sobre el "cómo" está argumentando. Por lo tanto, los rasgos distintivos que se señalarán a continuación no deben ser necesariamente actos conscientes.

"IRREFUTABILIDAD": LA VIOLENCIA ECLIPSADA

La pretensión de Objetividad es el motor principal del pensamiento único (antesala del fanatismo). En el espacio de una trifulca, nada puede ser más aplastante que demostrar que el argumento defendido está basado en evidencia neutral, concluyente y absoluta. Tal pretendida solvencia aumenta las chances de ganar la discusión: ansiedad de certeza y de dominación son una dupla peligrosamente potente.

Es obvio que, comparada con el uso de la fuerza bruta, ese tipo de argumentación se ve como inofensiva; pero está muy lejos de serlo: la compulsión a proteger la creencia propia cancela la deliberación, erradica lo distinto y anula al interlocutor en su 
alteridad. Este último sólo es epistémicamente valorado en tanto acepte las razones "correctas" que el argumentador violento defiende a capa y espada. La relación que entonces se busca instaurar es de estilo pedagógico-adoctrinante: "el que sabe" espera transmitirle la verdad al que la ignora, con lo cual se coloca en una posición up dentro de la relación. Su punto de vista será celosamente protegido, pero no como mero "punto de vista", sino como una verdad universal, que cualquiera en su "sano juicio" estaría dispuesto a aceptar. Tal arrogancia epistémica es la expresión de una manera de creer, desear, sentir y actuar bajo el mecanismo de un desprecio activo por todo aquello que no pertenece al espacio de la propia validación. El argumentador violento no necesita gritar, ni exasperarse, ni dar un puñetazo sobre la mesa: su fuerza reside en un exceso de autoafirmación que se complementa con un exceso de devaluación de todo aquello que no encaja en su marco de conocimiento aceptado. En su círculo social, tenderá a rodearse de aquellos que no cuestionan su "superioridad", promoviendo, así, cierto sectarismo. Contra el menor atisbo de crítica, reaccionará con actitud combativa (aunque puede invitar a sus potenciales detractores a una engañosa búsqueda de la verdad única). Para ello, puede echar mano de un conjunto de argucias que le funcionen para salir airoso de un conflicto, blindando así su postura de todo posible "boicot" o ataque.

Tales recursos, que Pereda (1994) denomina "vértigos argumentales", sirven para inmunizar sus ideas y prolongarlas (sin "ceder" ni dejar lugar para el cambio). Su "vertiginosidad" no da tregua a la pausa reflexiva que permitiría el movimiento de creencias; tiene sobre el interlocutor un efecto "mareador" que arrastra inadvertidamente o que bien crea una atracción difícilmente resistible: abruma, aturde. Al darle a sus premisas el estatuto de "obviedades", coloca el debate en el nivel de los sobreentendidos (allí donde ya no es posible algún aprendizaje o ensanchamiento de su horizonte argumentativo). Persigue frenéticamente la uniformidad conceptual, es decir, que los otros terminen pensando igual (con lo cual, nadie piensa, sino que obedecen). Sin tener plena lucidez sobre ello, pero con un entusiasmo pujante, puede apelar a extravagantes simplificaciones que barren detalles y precisiones sutiles; o, por el contrario, puede también recurrir a tácticas de complicación que tengan como finalidad la distracción o desorientación del interlocutor y el bloqueo de la discusión. En cualquiera de los dos casos (hipersimplificación o hipercomplicación), los extremos no permiten una evaluación equilibrada y contextualizada de hasta dónde es adecuado, en tal situación, simplificar o complejizar.

Como bien advierte Pereda (1994), en el transcurso de una argumentación vertiginosa, el argumentador no reconoce, ni está dispuesto a reconocer, las parcialidades que son intrínsecas al acto de argumentar (lo que previamente se llamó "violencia interna"). Por ejemplo, no concederá que la información que maneja puede ser incompleta, errónea o multívoca (por el contrario, pretenderá que sus creencias son completas y correctas); tampoco reconocerá que, al argumentar, se estructura un relato desde cierto género narrativo, que bien pudiera ser victimizante, acusatorio, catastrofista, frivolizante, entre otros. Para el argumentador vertiginoso, su relato no tiene 
un "estilo", ya que es una transparente "crónica de lo real", "totalmente verídica". Asimismo, aunque focaliza su atención únicamente sobre lo que pondera como "relevante" (e ignora lo "irrelevante"), considerará que esa relevancia es "natural", y no selectiva.

Si acaso se le señalaran estos sesgos con el propósito de mejorar la calidad de la discusión, el vertiginoso podría volver a resbalar: al intentar corregir su argumentación, volvería a argumentar con renovada vertiginosidad ("más de lo mismo"), tratando de depurar un sesgo con otro sesgo, ad infinitum. El vértigo le haría sucumbir a la tentación de mostrarse intransigente con relación a lo que no considera que abone a su propia opinión (que ni siquiera es vista como opinión). Aceleradamente negará o desestimará cualquier potencial señalamiento en contra de sus sesgos o parcialidades, pero, al hacerlo, podrá volver a caer -aunque sea involuntariamente- en un aumento de la parcialidad, enredando a todos en una argumentación cada vez más vertiginosa (Pereda, 1994: 331). En un escenario semejante, la intención de mantener viva la discusión sólo tiene como ideal la autoconfirmación; lo que hay detrás de la propia postura, en realidad no importa, porque "no existe".

Cuando la argumentación es tratada exclusivamente en su dimensión lógica, los aspectos caracterológicos de los argumentadores parecen ser superfluos. Pero, en cambio, cuando el foco de atención se deposita sobre los efectos prácticos de la argumentación, esos rasgos caracterológicos no pueden pasar desapercibidos; hay virtudes y vicios epistémicos que pueden, respectivamente, mejorar o debilitar la fuerza de una disputa. Una conversación que comenzó serenamente puede ir "subiendo de tono" hasta precipitarse hacia una argumentación vertiginosa, que no sólo supone cambios sobre los argumentos esgrimidos, sino también, y fundamentalmente, cambios en la manera de posicionarse ante el Otro. Este punto es especialmente pertinente para abandonar la falsa creencia de que la argumentación consiste en un aséptico intercambio de razones. Las decisiones que, para ser tomadas, nos ponen en la necesidad previa de argumentar, no dependen únicamente de lo que el argumentador afirma (que puede ser verdadero, falso o indeterminable), sino fundamentalmente de la confianza que depositamos sobre él como sujeto epistémico. Requerimos información acerca de quién da la información: lo que el otro dice, es escuchado en un determinado marco de credibilidad. Es decir, el significado de las palabras proferidas por el interlocutor lo interpretamos a la luz de la relación de fiabilidad que nos vincula con él.

Por supuesto que, el hecho de no advertir, ni explicitar, el tipo de relación que define cómo descifrar y sopesar los argumentos intercambiados, no evita que dicha relación sea el contexto dentro del cual se construyen nuestras reacciones argumentales ante los demás. La idea de que la calidad argumentativa no tiene nada que ver con quiénes son los argumentadores, es tan ilusoria como la de sostener que se argumenta exclusivamente con el lenguaje enunciativo verbalizado. Por el contrario, se argumenta "con todo el ser". Pereda lo sentencia crudamente: “¿Qué más tranquilizador, cuando 
no se sabe cómo actuar o qué pensar o decir, que fruncir el ceño y poner cara de asco?" (1999: 20).

\section{EPISTEMICIDIO: LA VIA REGIA DE LA ARROGANCIA}

En la base de una actitud de autosuperioridad epistémica reside un tipo de racionalidad que desconoce, desdeña o niega la validez de otro tipo de saberes que no encajen dentro del corsé del "pensamiento único". Esa razón anuladora, que ha sido bautizada por Pereda (1999) como "razón arrogante", está modelada por el universalismo filosófico que menosprecia lo diferente. Cuando se entabla una discusión, se tiende a querer decidir cuál de las versiones existentes es "la correcta y definitiva" (supuestamente, esa versión que cualquiera en su "sano juicio" tendría que aceptar); en ese punto, puede arrancar una competencia feroz de argumentos y contraargumentos, dando por sentado que, si hay dos explicaciones distintas de la misma situación, y una de ellas es "correcta", entonces la otra (o las otras) debe (o deben) estar necesariamente equivocada(s). Este principio tan básico no es sino la expresión de la ley de tercero excluido (un pilar de la lógica clásica: si una teoría es verdadera, su opuesta tiene que ser falsa, y no hay una tercera posibilidad). Y si bien la pretensión objetivista ha sido dominante en el terreno de la filosofía y la ciencia, también lo ha sido en las formas de conversación e interacción de la vida social cotidiana. Para quien está convencido de que su postura ES la correcta, no será difícil "deducir" que el otro, si discrepa, está equivocado. La interlocución con ese "Otro equivocado" solo puede tener el sentido de mostrarle su error, colonizar su campo argumental y "ayudarlo" a rectificar su idea para que pueda evidenciar "los hechos tal como son".

Así, quien detenta la pretensión de poseer un saber superior que no admite disonancia ni crítica, invalida cualquier diferencia; actúa de manera epistemicida (Santos, 1998: 208). El epistemicidio -muerte de todo saber alternativo- puede ocurrir no sólo refutando lo diferente, sino ignorándolo, tratándolo como si no existiera. En cierto modo, se asumiría una jerarquía "natural" de saberes cuya estratificación se daría por sentada. Funcionaría, en el ámbito del conocimiento, como una especie de "racismo epistémico" mediante el cual se inferioriza la diferencia, pero sin que se perciba como inferiorización (Santos, 2014: 21-66). Se niega, dentro del vínculo, la simultaneidad maduracional de los interlocutores (en el sentido de que, quien se autoconsidera más "avanzado", se ubica a sí mismo en un tiempo "progresivo"). A ese "Otro" con quien discute sólo se lo reconoce como un simultáneo en el espacio, pero no en el tiempo (Díaz, 2017: 7). Para ser un simultáneo pleno, ese "Otro" tendría que haber "evolucionado", lo cual significaría llegar a pensar del modo "correcto", andar el camino que le falta.

\section{LA INVALIDACIÓN ARGUMENTAL COMO UNA FORMA DE DESONTOLOGIZACIÓN}

Existe una delgada línea entre la desvalorización epistémica y la desvalorización ontológica: el Otro es menos porque sabe menos, y sabe menos porque es menos. En un 
escenario violento, no se "ningunea" sólo el conocimiento del Otro, sino su persona. Parafraseando a Gregory Bateson (1979: 30), el intolerante presenta el mapa como si fuese EL territorio. En la efervescencia de su argumentación objetivista, deviene un militante que se abraza a sus premisas como si fuesen dogmas; incluso podría apelar a un discurso democrático si con ello aumenta la adhesión del adversario y las posibilidades de salir triunfante "con legitimidad". Que le sea negada la razón, podría ser entendido como una incapacidad, un insulto, una ofensa o un maltrato; el disenso se toma como personal, ya que se discute para ganar, no para deliberar.

Ese estilo argumental conlleva a una conversación parasitaria; no resulta una práctica en la cual los participantes compartan el propósito de construir, juntos, una trama fundamentadora hacia una toma de decisión consensuada. No hay un camino a aperturar porque, a priori, ya hay una postura tomada con respecto a la diferencia. Para que la argumentación pudiera orientarse hacia la deliberación, sería necesaria cierta inocencia, entendida como la disposición a abrirse a la discordancia, a dejarse influir y a transformarse durante el acto conversacional. Si no es así, la argumentación no puede tener un sentido deliberativo. El rumbo de la conversación argumental se va trazando a medida que los partícipes lo van construyendo, y tiene una naturaleza inevitablemente situada (Gensollen, 2012: 120). Esto significa que, en la práctica, no sólo importa que un argumento sea correcto, sino que sea relevante; pero, justamente, la relevancia es un valor profundamente moldeado por las circunstancias específicas en las que se da el acto argumental. De modo que el proceso de argumentación está atravesado por condiciones cambiantes, dinámicas, siempre en movimiento. Ninguna idea o razón es intrínsecamente relevante, sino a la luz de expectativas e intereses válidos en ese momento particular (intereses que pueden coincidir, o no).

Por supuesto que, en una conversación en la que se adopta una postura vertiginosa, hay una gran insensibilidad hacia las necesidades y prioridades del entorno relacional (ya que la única urgencia sentida es la de salvaguardar los intereses personales o del grupo al cual se representa). Dado que el argumentador vertiginoso no admite un oyente activo, cancela la viabilidad de un diálogo, sustituyéndolo, a lo más, por una suma de monólogos. El Otro no es Otro, sino prolongación del sí mismo epistémicamente soberano, amo y esclavo de una "voz única" que se repite compulsivamente como eco de sí misma.

En cambio, cuando el encuentro conversacional se cimenta sobre bases dialógicas, emerge lo que Bajtín (1975/1981) entiende como "polifonía", ese momento en que una multiplicidad de voces independientes, no fundidas entre sí y totalmente válidas, afloran en el transcurso de un proceso de habla y escucha que evoluciona sincronizadamente, en un entrelazamiento de respuesta, anticipación y contestación que está vivo, es recíproco, va y viene. En el diálogo polifónico hay múltiples centros de subjetividad, o voz y agencia, sin que ninguno de ellos sea dominante; dialogar constituye un tipo de proceso comunicativo que, además de intelectual, es emocional y creativo; es un "dejarse llevar" por la conversación, sin intentar dirigirla excesivamente. Los hablantes 
se permiten a sí mismos estar presentes y vulnerables en un momento vivenciado y sentido, y participan en una experiencia nueva y común que, aun cuando es compartida, sigue siendo inexorablemente polisémica.

\section{LA CUESTIÓN DE LA “RACIONALIDAD” EN UNA CULTURA ARGUMENTAL}

Hay una dualidad bastante paradójica en torno a la argumentación: por un lado, se admite que argumentar es la vía menos violenta de resolver un conflicto. Pero, al mismo tiempo, se ha usado frecuentemente la metáfora de la guerra para caracterizar a la estructura de una conversación argumentada (como el debate, la disputa, la discusión, etcétera). Planteada desde tal metáfora, la argumentación supone una división binaria del campo discursivo entre proponente y oponente, entre argumentador $y$ contraargumentador, entre vencedor y vencido. Los argumentos entran en competencia, los contrincantes atacan y contraatacan, hasta que, finalmente, la contienda queda clausurada a favor del argumentador más racional.

Ahora bien, ¿qué significa ser "racional"? Desde una perspectiva clásica (y monocultural), la racionalidad se define en términos de un conjunto de criterios precisos, fijos, homogéneos y generales que validan los fundamentos últimos del conocimiento. "Razonar correctamente" supone, entonces, "saber deducir", lo cual refiere a que la argumentación conduzca al terreno de la certeza y la conclusividad. Esta idea de la racionalidad está estrechamente asociada a la visión de la teoría de la argumentación como estudio de la conexión lógica entre enunciados. Pereda (1994) la ha denominado "razón austera" precisamente porque ciñe el concepto de argumentación a un embudo tan cerrado que se acaba desestimando como "irracional" todo argumento que no sea concluyente, es decir determinado (deductivo).

Esta cosmovisión forma parte de una política conceptual hegemónica, que desvincula a la argumentación del ámbito de la vida cotidiana, en el que prima la incertidumbre y el uso de argumentos no-deductivos. Justo por eso Pereda propone una política epistémica diferente, la cual flexibiliza, amplia y diversifica el ámbito de la racionalidad. Cuestiona el sesgo simplificador fundamentalista, según el cual hay saber si y sólo si hay fundamentos indubitables. Si la cuestión de la argumentación se redujera al estudio de las reglas inferenciales y los argumentos deductivos, no tendría sentido alguno el planteamiento de la pragmática argumental. Los argumentos deductivos, si bien tienen un carácter necesario y son fácilmente mecanizables, se aplican cuando la argumentación ya se realizó, es decir, cuando la cuestión ya se cerró y sólo resta derivar una conclusión (1994: 39). La validez de la conclusión está autocontenida en las premisas del argumento mismo, no hay nada que esté "irresuelto", "abierto", ni que dependa de otros saberes externos (Harada, 2010).

Pero, en la práctica argumental, los argumentos no son estrictamente determinados. Como ya se dijo, no es suficiente con que sean formalmente válidos, o con que tengan un apoyo empírico fuerte: el tema de su pertinencia es primordial, sobre todo cuando lo que se busca es resolver un problema (Pereda, 1996: 12; Harada, 2010). Reconocer la 
consistencia lógica, los cuantificadores, las funciones de verdad, las tautologías, las contradicciones, las contingencias proposicionales y muchos otros conceptos claves de la lógica formal, no completa el kit que se necesita para un desempeño argumental generativo. Para la lógica formal, por ejemplo, una de las más graves amenazas del "pseudorazonamiento" sería el de cometer una falacia (un razonamiento inválido de apariencia intencional válida). Sin embargo, desde el punto de vista práctico, cometer un vértigo argumental puede tener efectos más devastadores que cometer una falacia. Un argumento podría ser formalmente correcto (válido), pero vertiginoso, lo cual llevaría a contaminar a toda la conversación (que contiene varios argumentos), forzaría el convencimiento, "desaparecería" al interlocutor e ignoraría que todo eso está ocurriendo (por lo cual sería mucho menos probable que se detecte el vértigo y se lo revise con ánimo de rectificación). Por el contrario, una argumentación podría estar apoyada en razonamientos inductivos o analógicos, y tener, no obstante, un alto potencial para lograr un acuerdo.

Esta elastización de la racionalidad invita a concebir un concepto de razón que tiene muy en cuenta los intereses perseguidos por los argumentadores, así como el tipo de asunto tratado, pero también induce a meditar sobre los diversos modos en que funciona la razón a lo largo de un ciclo argumental, en el cual, además de importar los apoyos y las reglas de inferencia, importan las virtudes, los vicios (vértigos) y las formas en que todo esto se articula (Pereda, 1996; Harada, 2010; Gensollen, 2014).

Pereda sostiene que hay ciertas reglas prudenciales que orientan el juicio, y sin las cuales no se dan las condiciones para una argumentación sana (no vertiginosa). Hay que recordar que la argumentación vertiginosa arrastra a la parcialidad (el empecinarse en un enfoque único), invalidando la diferencia. Para que tales "patologías" de la argumentación se puedan prevenir, no alcanza con aprender lógica formal (deductiva), sino que es necesario el cultivo de las ya mencionadas virtudes epistémicas y reglas prudenciales que funcionan como sugerencias, advertencias y precauciones teñidas de cautela sobre lo que podría empañar, descarriar o incluso arruinar un proceso argumental.

El virtuosismo epistémico tiene una ineludible dimensión ética, e incluso política, dado que aboga por la idea de que tales recomendaciones son fértiles e indispensables para promover la deliberación, mientras que su descuido y abandono arrastran las conversaciones muy afuera de las aspiraciones deliberativas. Por ejemplo, una regla prudencial muy importante advierte de "no sucumbir a la tentación de certeza o ignorancia, pero tampoco a la de poder o impotencia" (Pereda, 1994: 9). Cuando, en efecto, se incurre en alguna de esas tentaciones, la argumentación sale sobrando: ni los dogmáticos, ni los escépticos, ni los poderosos, ni los impotentes necesitan argumentos: se sitúan más allá de ellos (Pereda, 1994: 9).

Está claro que el mencionado autor reafirma el ejercicio de una práctica argumental rigorista pero dúctil, que acepte la incertidumbre como parte de una lógica viva, una argumentación tentativa, complicada, antifundamentalista pero basada en una razón 
reflexiva (que integre el lenguaje figurado, la probabilidad, la historia de los conceptos, las metáforas, el quién dice una cosa y a quién) y enfática, es decir, competente para afrontar y resolver problemas teóricos y prácticos (Harada, 2010: 240-242).

Según Pereda, cada cultura (tradición, sociedad, institución, grupo) implementa (u obstruye) mecanismos o procedimientos públicos para producir situaciones argumentales, detectar dificultades, conflictos y problemas, así como medios de participación para remediarlos, adquiriendo "grados de racionalidad" que permitan evitar el uso de la fuerza bruta (1994: 322; Harada, 2010: 242).

Hay que considerar que esta apreciación de una cultura argumental p/uralista no implica la quimera de que en todo momento los individuos abordarán sus diferencias a través de la argumentación. Ciertamente, la formación de virtudes epistémicas y reglas prudenciales sería un gran facilitador de la crítica y la deliberación, pero, ante determinadas coyunturas violentas, podría no haber condiciones mínimas para el despliegue de ciclos argumentales. No obstante, en el momento en que se pretendiera legitimar esa violencia, se terminaría apelando a la argumentación, aunque pudiera tratarse de una argumentación infectada de esas tentaciones o excesos que obnubilan la capacidad de juicio. Aquí habría que subrayar que esa violencia argumental vertiginosa -internamente desencadenada- podría intensificar la violencia externa, moviendo una espiral cada vez más destructiva.

\section{MÁS ALLÁ DEL PARASITISMO: HACIA UNA "SALUD ARGUMENTATIVA"}

Dentro de los múltiples componentes de un proceso argumental sano, los conocimientos (teoría de la argumentación) se articulan con las habilidades (las técnicas procedimentales) y las actitudes (rasgos de carácter, disposiciones). La idea central es que las disputas estén reguladas desde adentro (es decir, desde la propia argumentación), y no desde afuera, es decir, desde el control externo de criterios rígidos que sólo serían ataduras argumentales (Pereda, 1994: 14; Harada, 2010: 237). Así, el "buen argumentar" no es sólo cuestión de saber cómo apoyar con razones, sino saber cómo organizar una discusión, cómo aceptar consecuencias, saber cómo clarificar el discurso, cómo identificar la estructura del argumento, saber cuándo la evidencia es suficiente e insuficiente, y cómo buscar alternativas (Morado, 2009; Gensollen, 2012). Asimismo, es importante visibilizar las creencias presupuestas en la argumentación, y decantar cuáles son innecesarias si el objetivo es resolver un conflicto y llegar a un acuerdo. Un buen argumentador también debe ser capaz de vislumbrar las presunciones que se siguen de lo acordado, e identificar qué acciones están incluidas dentro del compromiso adquirido si acepta los términos del acuerdo (Gensollen, 2012: 124-125).

Otro aspecto medular en el proceso de argumentación es el de saber qué preguntar y también el de saber determinar si es factible o no embarcarse en una argumentación. Incluso Pereda señalará que el saber cuándo es adecuado afrontar un conflicto de creencias representa el primer paso de un ciclo argumental exitoso. En apoyo a este señalamiento, Gensollen recoge el consejo práctico de Aristóteles cuando advertía que 
"no hay que discutir con todo el mundo, ni hay que ejercitarse frente a un individuo cualquiera. Pues, frente a algunos, los argumentos se tornan necesariamente viciados" (2012: 115). Son, en efecto, los que parecen evitar el encuentro.

El virtuosismo epistémico en lo que respecta al contenido de los argumentos está dado, según Pereda, por la coherencia, la contrastabilidad empírica, el poder explicativo y el predictivo (virtudes procedimentales). En lo que concierne al agente argumentador, el virtuosismo tiene que ver con rasgos personales, de naturaleza epistémica pero también moral y política. Pereda las denomina virtudes morfológicas y se refieren a valores donde lo epistémico y lo ético se hibridan (como ocurre con la integridad, que implica autonomía intelectual, o con el rigor, que supone justeza intelectual). De tales virtudes, destacan las siguientes (Gensollen, 2012: 125-126):

a) Buena voluntad: estar dispuesto a afrontar argumentativamente un conflicto de creencias, a hacerse entender y entender a su interlocutor, así como a aceptar las consecuencias de la discusión argumentada.

b) Caridad intelectual (o "espíritu de rescate"): suponer máxima racionalidad y coherencia en su interlocutor. Ello se traducirá en la disposición a reconstruir de la mejor manera las razones del opositor.

c) Falibilidad: asumir la posibilidad de que cualquiera de las propias creencias mantenidas en el debate podría ser falsa, incompleta, sesgada, revisable.

d) Coalescencia: priorizar el acuerdo y la solución del conflicto, antes que la verdad o la victoria en la disputa.

e) Pluralismo: apreciar las diferencias y aprender de ella, tal como hace el cosmopolita (sin esperar la convergencia en una única cosmovisión, en un único modo de vida o en un único modo de hacer las cosas).

Estas virtudes argumentales son adquiribles mediante su labranza dentro de un contexto que les sea favorable, esto es, dentro de un medio en el cual la argumentación sea entendida como un preámbulo del ejercicio de deliberación que anteceda a la acción colectiva. Por ello mismo, la pregunta por el cómo argumentar remite a la pregunta de qué tipo de vida social, política y cultural se desea fomentar.

\section{CONCLUSIONES}

La capacidad de "dar razones" es distintiva de la condición humana. Al argumentar, se crean mundos, mapas, y también relaciones sociales. En el acontecer de la vida común, la argumentación no siempre tiene como objetivo principal el descubrir "la verdad"; en una gran cantidad de situaciones, se busca llegar a un acuerdo para resolver un asunto. A lo largo de ese proceso, la argumentación transcurre como una experiencia emocional que mueve los vínculos en direcciones cambiantes.

La concepción de que la argumentación está inexorablemente unida al ideal de Objetividad, a la lógica deductiva, al pensamiento rigorista y a la certeza obstaculiza la comprensión de los complejos modos en que se usa la argumentación en los encuentros interpersonales habituales. No considerar el contexto en el cual se sitúan los 
respectivos agentes argumentativos, ni sus particularidades caracterológicas, sería tan restrictivo como evaluar sus argumentos ponderando sólo dichas características personales. Ninguno de esos dos extremos dejaría entrever el ensamblaje de conocimientos, habilidades y actitudes que se activan en los encuentros conversacionales cuando estos se dan dentro de un sistema social que alienta los procesos de deliberación (tal como ocurre en las auténticas democracias).

En tal sentido, tomar como foco de análisis la argumentación dialógica (ese intercambio que acontece entre dos o más personas) arroja luz sobre dinámicas relacionales que no son inteligibles cuando se aborda la argumentación como un mero ejercicio lógico individual. Por otro lado, contemplando que las decisiones que se toman dependen en gran medida de la calidad de las deliberaciones realizadas, y que estas, a su vez, están muy influidas por el tipo de argumentación sostenida, puede decirse que la formación de una consciencia crítica sobre el propio estilo argumental es de máxima trascendencia para lograr autonomía intelectual. A la inversa, amurallar sistemáticamente la propia argumentación para eludir la crítica, instala un parasitismo epistémico mediante el cual el agente argumentador subsiste alimentándose de la autovalidación y de la convalidación ajena. Su racionalidad indolente (Santos, 2005: 152) lo vuelve peligrosamente irresponsable, ya que, como bien alertaron Cecchin, Lane y Ray,

una lealtad excesiva a una idea específica hace que la persona no sea responsable de las consecuencias morales inherentes a ella. Si sobreviene un desastre, el responsable no será el individuo, sino la Idea (con "I" mayúscula) que ha comandado la acción (2003: 26).

Esas ideas o prejuicios funcionan como puntos ciegos; de allí al pensamiento vertiginoso hay un deslizamiento casi natural, que desemboca en la cerrazón, la soberbia, la altivez, la intransigencia y la discriminación epistemicida. Como afirma Pereda (1999, portada), "La arrogancia desfigura los mejores pensamientos y pervierte hasta las prácticas más generosas".

\section{BIBLIOGRAFÍA}

Bateson, Gregory. (1979). Mind and Nature. New York, NY: Bantam Books.

Bakhtin, Mijaíl. (1975/1981). La imaginación dialógica. Austin: Holquist Ed. y Trad., University of Texas, TX.

Cecchin, Gianfranco., Lane, Gerry., Ray, Wendel. (2003). Irreverencia: una estrategia de supervivencia para terapeutas, Barcelona: Paidós. 
Díaz, Martín. (2017). Racismo epistemológico y occidentalocentrismo: apuntes para una descolonización de la tradición hegemónica del conocimiento. Revista de Epistemología y Ciencias Humanas, 9, mayo, pp. 1-18.

Gensollen, Mario. (2012). Virtudes argumentativas: hacia una cultura de la paz. Euphyía. VI-11, pp. 116-131.

Gensollen, Mario. (2014). Virtudes y vicios argumentativos: A veinte años de Vértigos argumentales, de Carlos Pereda. Tópicos, Revista de Filosofía. 47, pp. 159-195.

Harada, Eduardo. (2010). Carlos Pereda y la cultura argumentativa. Andamios, VII-14, pp. 225-262.

Illouz, Eva. (2009). El Consumo de la Utopía Romántica. El amor y las contradicciones Culturales del Capitalismo. Versión en español. Buenos Aires: Editorial Katz.

Morado, Raymundo. (2009). Compendio de lógica. México: Editorial Torres Asociados.

Pereda, Carlos. (1994). Vértigos argumentales. Una ética de la disputa. Barcelona: Anthropos \& UAM-Iztapalapa.

Pereda, Carlos. (1996). ¿Qué es un buen argumento? Theoria. Segunda Época, 11(25), San Sebastián: Universidad del País Vasco, pp. 7-20.

Pereda, Carlos. (1999). Crítica de la razón arrogante. México: Taurus-Alfaguara.

Santos, Boaventura de Sousa. (1998). La globalización del derecho: los nuevos caminos de la regulación y la emancipación, Bogotá, ILSA-Universidad Nacional de Colombia.

Santos, Boaventura de Sousa (2005). El milenio huérfano. Ensayos para una nueva cultura politica, Madrid: Trotta/ILSA.

Santos, Boaventura de Sousa y Meneses, María Paula. (Eds.) (2014). Epistemologías del Sur. Perspectivas. Madrid: Akal.

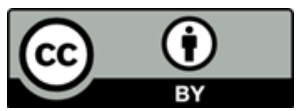

